\section{Role of Primary-Site Surgery in Stage IV Breast Cancer}

\section{DEAR EDITOR:}

We appreciate the comments from surgeons of the University of Ionnina in Greece regarding our report concerning the role of primary-site surgery in stage IV breast cancer. ${ }^{1,2}$ We agree that a randomized trial to definitively answer the question is not worth doing for financial, practical, and ethical reasons. For instance, how would you randomize a woman with a bleeding fungating advanced local breast cancer with metastases when individual judgment regarding palliation is the critical issue? How would you randomize a woman with an oligometastatic lung metastasis with surgically manageable breast cancer? There are too many other issues in breast cancer control more pressing than this. Our retrospective matched-pair analysis was deliberately chosen as the most practical, rapid, and inexpensive method to obtain a reasonable evidence-based answer to the question of the role of primary-site surgery in stage IV breast cancers. This revealed that case selection bias accounted for most, if not all, of the apparent survival advantage by resection of the primary-site cancer.

It is important to recognize our subsidiary finding, however, which was to demonstrate the lack of a causal relationship between local primary-site control success and long-term survival. We demonstrated directly the selection bias for local breast surgery based on the biological behavior of the cancer, which results in the coincidence between successful local control efforts and survival. Survival depends on the biological aspects of the metastatic disease, i.e., timing of clinical metastatic appearance (early versus late), organ specificity (bone versus liver), estrogen receptor analysis (positive versus negative), number of metastases (few versus many), distribution (oligometastases versus multiple organ metastases), and initial systemic therapeutic results (responsive versus nonresponsive), but not what surgical approach is undertaken for the primary breast cancer.

To address another of the letter writers' concerns, we have proposed that the best prevention strategy for invasive breast cancer is detection by screening mammography, with resection of preinvasive ductal carcinoma in situ (DCIS) when discovered. ${ }^{3,4}$ As with polyp removal that prevents colorectal cancer, dysplasia and in situ treatments that prevent invasive cervical cancer, and dysplasia and in situ melanoma treatment that prevents invasive melanoma, removal of DCIS prevents many, if not most, later invasive ductal carcinomas of the breast. Emphasis on this neglected mechanism of prevention has been of little avail or acceptance, as the breast cancer community, and indeed the letter writers themselves, ignore this logical disease sequence and prevention strategy. The recent significant decline in breast cancer incidence in the USA is consistent with our thesis in both timing and magnitude. The incidence decline began about the year 2000, 15 years after the upsurge in screening mammography in the mid 1980s, and has continued. We do not accept their attribution of declining incidence to breast magnetic resonance imaging (MRI) or genetic screening; we also do not attribute the continued steady incidence decline to cessation of postmenopausal use of hormones or the use of Tamoxifen for prevention in the small proportion of high-risk women. The magnitude and timing of the incidence decline might well include contributions of these other proposed mechanisms, but the year after year successive surgical removal of the preinvasive DCIS commonly detected seems the most logical in producing the continuously declining incidence of invasive ductal carcinomas, the largest component, by far, of invasive breast cancers.

\section{Blake Cady, MD}

Boston, MA

e-mail: bcady123@comcast.net

Published Online: 18 February 2009

(C) Society of Surgical Oncology 2009

\section{REFERENCES}

1. Ignantiadou E, Ziogas D, Fatouros M. Stage IV breast cancer-is surgical resection of clinical utility? Ann Surg Oncol. 2009. DOI: 10.1245/s10434-008-0312-5.

2. Cady B, Nathan NR, Michaelson JS, Golshan M, Smith BL. Matched pair analyses of stage IV breast cancer with or without resection of primary breast site. Ann Surg Oncol. 2008;15:3384-95.

3. Cady B. How to prevent invasive breast cancer: detect and excise duct carcinoma in situ. J Surg Oncol. 1998;69:60-2.

4. Cady B, Chung, MA. The prevention of invasive breast carcinoma. Cancer. 2004;101(10):2147-1. 